PROCEEDINGS OF THE

AMERICAN MATHEMATICAL SOCIETY

Volume 127, Number 9, Pages 2671-2678

S 0002-9939(99)04805-4

Article electronically published on March 16, 1999

\title{
OPIAL'S MODULUS AND FIXED POINTS OF SEMIGROUPS OF MAPPINGS
}

\author{
TADEUSZ KUCZUMOW
}

(Communicated by Palle E. T. Jorgensen)

\begin{abstract}
If $X$ is a Banach space with the non-strict Opial property and $r_{X}(1)>0$ and $C$ is a nonempty convex weakly compact subset of $X$, then every semigroup $\mathfrak{T}=\left\{T_{t}: t \in G\right\}$ of asymptotically regular selfmappings of $C$ with $\sigma(\mathfrak{T})<1+r_{X}(1)$ has a common fixed point.
\end{abstract}

\section{InTRODUCTION}

The study of fixed points of uniformly lipschitzian mappings was initiated by K. Goebel and W. A. Kirk in 1973 [10] and next developed by several authors (see [4], [6], [12], [13], [18] and [20] for updated literature). Recently T. Dominguez Benavides and H.-K. Xu [8] introduced a new geometrical coefficient which is especially useful in the investigation of asymptotically regular lipschitzian mappings [6], [13]. The aim of this paper is to improve the known fixed point theorems for this class of mappings in Banach spaces with the non-strict Opial property.

\section{Preliminaries}

Throughout $(X,\|\cdot\|)$ is a Banach space which is assumed not to be Schur. That is, $X$ has weakly convergent sequences that are not norm convergent. We will use the following notations. If $\left\{x_{n}\right\}_{n \geq 1}$ is a bounded sequence in $(X,\|\cdot\|)$ and $x \in X$, then $r_{a}\left(x,\left\{x_{n}\right\}\right)=\limsup _{n \rightarrow \infty}\left\|x-x_{n}\right\|$. A number $\operatorname{diam}_{a}\left\{x_{n}\right\}=$ $\lim _{k \rightarrow \infty} \sup \left\{\left\|x_{n}-x_{m}\right\|: n, m \geq k\right\}$ is called an asymptotic diameter of $\left\{x_{n}\right\}$. A weakly convergent sequence coefficient (see [5] is defined by

$$
W C S(X)=\sup \left\{k: k \cdot \limsup _{n \rightarrow \infty}\left\|x_{n}\right\| \leq \operatorname{diam}_{a}\left(\left\{x_{n}\right\}\right)\right.
$$

for all weakly convergent to 0 sequences in $X\}$.

Received by the editors February 27, 1997 and, in revised form, September 25, 1997 and November 14, 1997.

1991 Mathematics Subject Classification. Primary 47H10, 46B20.

Key words and phrases. Opial's modulus, semigroups of mappings, fixed points.

(C)1999 American Mathematical Society 
A Banach space $X$ is said to satisfy Opial's condition [24] (a non-strict Opial condition), if whenever a sequence $\left\{x_{n}\right\}$ in $X$ converges weakly to $x$, then

$$
\begin{gathered}
\liminf _{n \rightarrow \infty}\left\|x-x_{n}\right\|<\liminf _{n \rightarrow \infty}\left\|y-x_{n}\right\| \\
\left(\liminf _{n \rightarrow \infty}\left\|x-x_{n}\right\| \leq \liminf _{n \rightarrow \infty}\left\|y-x_{n}\right\|\right)
\end{gathered}
$$

for every $y \in X \backslash\{x\}$.

Opial's modulus $r_{X}$ of $X$ is introduced (in [25]) as

$$
r_{X}(c)=\inf \left\{\liminf _{n \rightarrow \infty}\left\|x_{n}+x\right\|-1\right\},
$$

where $c \geq 0$ and the infimum is taken over all $x \in X$ with $\|x\| \geq c$ and all sequences $\left\{x_{n}\right\}$ in $X$ such that $w-\lim _{n \rightarrow \infty} x_{n}=0$ and $\liminf _{n \rightarrow \infty}\left\|x_{n}\right\| \geq 1$. The function $r_{X}$ is continuous and nondecreasing [23], [28]. Next we have the following inequality (see [23]):

$$
1+r_{X}(1) \leq W C S(X) .
$$

A semi-Opial coefficient with respect to the weak topology, $w$-SOC $(X)$ for short, is defined as follows (see [3]):

$$
w-S O C(X)=\sup \left\{k: k \cdot \inf _{\left\{x_{n_{i}}\right\}_{i \geq 1}, x_{n_{i}} \rightarrow y} r_{a}\left(y,\left\{x_{n_{i}}\right\}_{i \geq 1}\right) \leq \operatorname{diam}_{a}\left(\left\{x_{n}\right\}\right)\right.
$$

for each $\left\{x_{n}\right\}_{n \geq 1}$ with weakly compact $\overline{\operatorname{conv}}\left(\left\{x_{n}\right\}_{n \geq 1}\right)$ and $\left.x_{n}-x_{n+1} \stackrel{n}{\rightarrow} 0\right\}$.

If $w$-SOC $(X)>1$, then $(X,\|\cdot\|)$ has a uniform semi-Opial property with respect to the weak topology [3]. Directly from the above definitions we get [3]

$$
1 \leq W C S(X) \leq w-S O C(X) .
$$

As we mentioned in the Introduction T. Domínguez Benavides and H.-K. Xu defined the constant $\kappa_{\omega}(X)$ in Banach spaces [8]. If $X$ is a Banach space and $C$ is a nonempty convex bounded closed subset of $X$, then

(a) a number $b \geq 0$ has a property $\left(P_{\omega}\right)$ with respect to $C$ if there exists $a>1$ such that for all $x, y \in C$ and $r>0$ with $\|x-y\| \geq r$ and each weakly convergent sequence $\left\{z_{n}\right\}$ with elements in $C, \lim \sup _{n \rightarrow \infty}\left\|x-z_{n}\right\| \leq$ ar and $\limsup _{n \rightarrow \infty}\left\|y-z_{n}\right\| \leq b r$, there exists $z \in C$ such that $\liminf _{n \rightarrow \infty}\left\|z-z_{n}\right\|$ $\leq r$

(b) $\kappa_{\omega}(C)=\sup \left\{b>0: b\right.$ has the property $\left(P_{\omega}\right)$ with respect to $\left.C\right\}$;

(c) $\kappa_{\omega}(X)=\inf \left\{\kappa_{\omega}(C): C\right.$ is a nonempty bounded convex closed subset of $\left.X\right\}$.

It is easy to observe that

$$
\begin{aligned}
\kappa_{\omega}(X)= & \inf \left\{\kappa_{\omega}(C): C\right. \text { is a nonempty bounded convex } \\
& \text { weakly compact subset of } X\} .
\end{aligned}
$$

Hence we get $\kappa_{\omega}(X) \leq W C S(X)[8]$.

Let $X$ be a Banach space and $T: C \rightarrow C$. We use a symbol $|T|$ to denote the exact Lipschitz constant of $T$, i.e.,

$$
|T|=\inf \{k \in[0, \infty]:\|T x-T y\| \leq k\|x-y\| \text { for all } x, y \in C\} .
$$


Let $X$ be a Banach space, $C$ a closed convex bounded nonempty subset of $X, G$ an unbounded subset of $[0, \infty)$ such that

$$
\begin{gathered}
t+h \in G \text { for all } t, h \in G, \\
t-h \in G \text { for all } t, h \in G \text { with } t \geq h
\end{gathered}
$$

and $\mathfrak{T}=\left\{T_{t}: t \in G\right\}$ a family of self-mappings on $C$. $\mathfrak{T}$ is called a semigroup of mappings on $C$ if

(i) $T_{s+t} x=T_{s} T_{t} x$ for all $s, t \in G$ and $x \in C$,

(ii) for each $x \in C$, a mapping $t \rightarrow T_{t} x$ from $G$ into $C$ is continuous when $G$ has the relative topology of $[0, \infty)$

If $\mathfrak{T}$ satisfies (i) - (ii), then

$$
\sigma(\mathfrak{T})=\liminf _{t \rightarrow \infty}\left|T_{t}\right|
$$

If $\mathfrak{T}$ satisfies (i) - (ii) and

$$
\sup _{t}\left|T_{t}\right|=k<\infty,
$$

then $\mathfrak{T}$ is called a uniformly lipschitzian (uniformly $k$-lipschitzian) semigroup.

The simplest uniformly lipschitzian semigroup is a semigroup of iterates of a mapping $T: C \rightarrow C$ with

$$
\sup _{n}\left|T^{n}\right|=k<\infty .
$$

In this case $T$ is called a uniformly $k$-lipschitzian mapping. In a natural way this kind of semigroup appears in the problem of stability of the fixed point property for nonexpansive mappings. Namely, if $(X,\|\cdot\|)$ is a Banach space, $\|\cdot\|_{1}$ is an equivalent norm such that the Banach-Mazur distance

$$
\begin{gathered}
d\left((X,\|\cdot\|),\left(X,\|\cdot\|_{1}\right)\right) \\
=\inf \left\{\|f\|\left\|f^{-1}\right\|: \quad f: X \rightarrow X \text { is an isomorphism }\right\}=k<\infty
\end{gathered}
$$

and $T: C \rightarrow C$ is $\|\cdot\|_{1}$-nonexpansive, then $T$ is uniformly $k$-lipschitzian in the norm $\|\cdot\|$. In the Hilbert space $l^{2}$ J.-P. Baillon [1] found an example of a fixedpoint-free uniformly $\frac{\pi}{2}$-lipschitzian mapping and D. Tingley [26] proved that any noncontractive (i.e.

$$
\|x-y\| \leq\left\|T^{n} x-T^{n} y\right\|
$$

for $x, y \in C$ and $n=1,2, \ldots)$ uniformly $k$-lipschitzian mapping with $k<\frac{\pi}{2}$ acting on a closed bounded set in the Hilbert space has a common fixed point. The results obtained in [7], [16], [17] and [22] give a new light on the Baillon example. They imply that there does not exist an equivalent norm in $l^{2}$ with the Banach-Mazur distance less or equal to $\frac{\pi}{2}$ in which the Baillon mapping is nonexpansive.

In [14] one also can find the interesting construction of the uniformly lipschitzian mapping with

$$
\liminf _{n \rightarrow \infty}\left|T^{n}\right|<\limsup _{n \rightarrow \infty}\left|T^{n}\right| .
$$

If $\mathfrak{T}$ satisfies (i) - (ii) and for each $x \in C, h \in G$

$$
\lim _{t \rightarrow \infty}\left\|T_{t+h} x-T_{t} x\right\|=0,
$$


then $\mathfrak{T}$ is asymptotically regular. The concept of asymptotic regularity is due to F. E. Browder and W. V. Petryshyn [2]. In [21] and [27] one can find two very interesting examples of asymptotically regular mappings without fixed points. By the Ishikawa result [15] (see also [9], [11] and [19]) in the problem of stability of the fixed point property for nonexpansive mappings in Banach spaces it is sufficient to consider asymptotically regular and nonexpansive mappings which become asymptotically regular and uniformly lipschitzian mappings in each equivalent norm to the original one.

In [6] and [8] the following theorem was proved (we leave it here in the form due to J. Górnicki [13]).

Theorem 2.1. Let $X$ be a Banach space, $C$ a nonempty convex weakly compact separable subset of $X$ and $\mathfrak{T}=\left\{T_{t}: t \in G\right\}$ be an asymptotically regular semigroup. If either

$$
\sigma(\mathfrak{T})=k<\sqrt{W C S(X)}
$$

or

$$
\sigma(\mathfrak{T})=k<\kappa_{\omega}(X),
$$

then there exists $z$ in $C$ such that $T_{t} z=z$ for all $t \in G$.

If we deal with the semi-Opial coefficient with respect to the weak topology, then the following theorem is valid [4].

Theorem 2.2. Let $X$ be a Banach space, $C$ a nonempty convex weakly compact subset of $X$ and $\mathfrak{T}=\left\{T_{t}: t \in G\right\}$ be an asymptotically regular semigroup of uniformly lipschitzian mappings, i.e. there exists a constant $k$ such that $\left|T_{t}\right| \leq k$ for every $t \in G$. If

$$
k<\sqrt{w-S O C(X)}
$$

then there exists $z$ in $C$ such that $T_{t} z=z$ for all $t \in G$.

In the next section we will try to improve the upper bounds of $\sigma(\mathfrak{T})$ which guarantee the fixed point property of $\mathfrak{T}$ in Banach spaces with the nonstrict Opial property. Our result is new even for the semigroups of iterates of uniformly lipschitzian mappings.

Finally let us observe that the class of uniformly lipschitzian mappings on $C$ is completely characterized as the class of mappings on $C$ which are nonexpansive relative to some metric on $C$ which is equivalent to the norm [12].

\section{EXISTENCE OF COMMON FIXED POINTS OF SEMIGROUPS OF MAPPING}

We begin with the following simple fact.

Lemma 3.1. If for $s>0$ and $c \geq 0$ we define $r_{X, s}(c)$ by

$$
r_{X, s}(c)=\inf \left\{\liminf _{n \rightarrow \infty}\left\|x_{n}+x\right\|-s\right\}
$$

where the infimum is taken over all $x \in X$ with $\|x\| \geq c$ and all sequences $\left\{x_{n}\right\}$ in $X$ such that $w-\lim _{n \rightarrow \infty} x_{n}=0$ and $\liminf _{n \rightarrow \infty}\left\|x_{n}\right\| \geq s$, then we get

$$
s+r_{X, s}(c)=s\left(1+r_{X}\left(\frac{c}{s}\right)\right) \text {. }
$$

The above lemma is used in the proof of our main result. 
Theorem 3.2. Let $X$ be a Banach space with $r_{X}(1)>0$ and with the nonstrict Opial property, $C$ a nonempty convex weakly compact subset of $X$ and $\mathfrak{T}=$ $\left\{T_{t}: t \in G\right\}$ an asymptotically regular semigroup with

$$
\sigma(\mathfrak{T})=k<1+r_{X}(1) .
$$

Then there exists $z$ in $C$ such that $T_{t} z=z$ for all $t \in G$.

Proof. Let us select a sequence $\left\{t_{n}\right\}$ and $0<c<1$ such that

$$
\begin{gathered}
\sigma(\mathfrak{T})=k=\lim _{n \rightarrow \infty}\left|T_{t_{n}}\right|, \quad t_{n} \rightarrow \infty, \\
\sigma(\mathfrak{T}) \leq \sup _{n}\left|T_{t_{n}}\right|<1+r_{X}(c)<1+r_{X}(1) .
\end{gathered}
$$

Since one can easily construct a nonempty closed convex separable subset $C_{0}$ of $C$ which is invariant under each $T_{t_{n}}$ (i.e. $T_{t_{n}}\left(C_{0}\right) \subset C_{0}$ for $\left.n=1,2, \ldots\right)$, we may assume for a while that $C$ itself is separable. By passing to a subsequence this makes possible to assume additionally that for each $x \in C$ the sequence $\left\{T_{t_{n}} x\right\}$ is weakly convergent and

$$
r_{a}\left(y,\left\{T_{t_{n}} x\right\}\right)=\lim _{n \rightarrow \infty}\left\|y-T_{t_{n}} x\right\|
$$

for every $x, y \in C$. Let us define a function $p$ on $C$ by

$$
p(x)=r_{a}\left(y,\left\{T_{t_{n}} x\right\}\right)=\lim _{n \rightarrow \infty}\left\|y-T_{t_{n}} x\right\|,
$$

where $T_{t_{n}} x \rightarrow y$. Then for $T_{t_{n}} x \rightarrow y$ we have

$$
p(y) \leq c p(x) .
$$

Let us suppose this were not the case. Then we can find sequences $T_{t_{n}} x \rightarrow y$ and $T_{t_{n}} y \rightarrow z$ such that (after eventually throwing out a finite number of indices - see the limit which appears in (3.2))

$$
\inf _{m}\left\|z-T_{t_{m}} y\right\|>c p(x) .
$$

If $p(x)=r\left(y,\left\{T_{t_{n}} x\right\}\right)=0$, then we have

$$
\begin{gathered}
\left\|y-T_{t_{m}} y\right\| \leq r_{a}\left(y,\left\{T_{t_{n}} x\right\}\right)+r_{a}\left(T_{t_{m}} y,\left\{T_{t_{n}} x\right\}\right) \\
\leq p(x)+\left|T_{t_{m}}\right| \cdot r_{a}\left(y,\left\{T_{t_{n}-t_{m}} x\right\}_{n}\right)
\end{gathered}
$$

and the asymptotic regularity of $\mathfrak{T}$ implies $\left\|y-T_{t_{m}} y\right\|=0$ for $m=1,2, \ldots$ and therefore $y=z$, but this contradicts (3.4). Hence $p(x)=r_{a}\left(y,\left\{T_{t_{n}} x\right\}\right)>0$. Now the asymptotic regularity of $\mathfrak{T}$, the nonstrict Opial property and the application of (3.1) and (3.2) yield to the following contradiction:

$$
\begin{gathered}
{\left[1+r_{X}(c)\right] \cdot p(x)=\left[1+r_{X}(c)\right] \cdot r_{a}\left(y,\left\{T_{t_{n}} x\right\}\right)>\sigma(\mathfrak{T}) \cdot r_{a}\left(y,\left\{T_{t_{n}} x\right\}\right)} \\
\geq \limsup _{m \rightarrow \infty} r_{a}\left(T_{t_{m}} y,\left\{T_{t_{n}} x\right\}\right) \geq \limsup _{m \rightarrow \infty}\left[1+r_{X}\left(\frac{\left\|y-T_{t_{m}} y\right\|}{p(x)}\right)\right] \cdot p(x) \\
=\left[1+r_{X}\left(\frac{\lim _{m \rightarrow \infty}\left\|y-T_{t_{m}} y\right\|}{p(x)}\right)\right] \cdot p(x) \\
\geq\left[1+r_{X}\left(\frac{\lim _{m \rightarrow \infty}\left\|z-T_{t_{m}} y\right\|}{p(x)}\right)\right] \cdot p(x) \geq\left[1+r_{X}(c)\right] \cdot p(x) .
\end{gathered}
$$


Hence the inequality (3.3) is valid. Thus we are able to construct the sequence $\left\{x_{m}\right\} \subset C$ in the following way:

$$
\begin{gathered}
x_{0} \in C \quad \text { arbitrary, } \\
x_{l}=w-\lim _{n \rightarrow \infty} T_{t_{n}} x_{l-1} \quad \text { for } l=1,2, \ldots .
\end{gathered}
$$

Using (3.3), the $w$-l.s.c. of the norm and the asymptotic regularity of $\mathfrak{T}$ we obtain

$$
\begin{gathered}
\left\|x_{l+1}-x_{l}\right\| \leq r_{a}\left(x_{l+1},\left\{T_{t_{n}} x_{l}\right\}\right)+r_{a}\left(x_{l},\left\{T_{t_{n}} x_{l}\right\}\right) \\
\leq p\left(x_{l}\right)+\limsup _{n \rightarrow \infty} \limsup _{m \rightarrow \infty}\left\|T_{t_{m}} x_{l-1}-T_{t_{n}} x_{l}\right\| \\
\leq p\left(x_{l}\right)+\limsup _{n \rightarrow \infty} \limsup _{m \rightarrow \infty}\left[\left\|T_{t_{m}} x_{l-1}-T_{t_{n}+t_{m}} x_{l-1}\right\|+\left\|T_{t_{n}+t_{m}} x_{l-1}-T_{t_{n}} x_{l}\right\|\right] \\
\leq p\left(x_{l}\right)+k \cdot p\left(x_{l-1}\right) \leq c^{l-1} \cdot(c+k) \cdot p\left(x_{0}\right)
\end{gathered}
$$

which shows that $\left\{x_{l}\right\}$ is strongly convergent to $\bar{x}$ and

$$
p(\bar{x}) \leq \lim _{l \rightarrow \infty} \lim _{n \rightarrow \infty}\left[\left\|\bar{x}-x_{l+1}\right\|+\left\|x_{l+1}-T_{t_{n}} x_{l}\right\|+\left|T_{t_{n}}\right| \cdot\left\|x_{l}-\bar{x}\right\|\right]=0 .
$$

The asymptotic regularity of $\mathfrak{T}$ implies $T_{t_{n}} \bar{x}=\bar{x}$ for $n=1,2, \ldots$. Now we return to the original set $C$ and the semigroup $\mathfrak{T}$. Then, if we apply again the asymptotic regularity of $\mathfrak{T}$ we get

$$
\left\|T_{t} \bar{x}-\bar{x}\right\|=\lim _{n \rightarrow \infty}\left\|T_{t+t_{n}} \bar{x}-T_{t_{n}} \bar{x}\right\|=0
$$

for each $t \in G$ and this completes the proof.

\section{Comparison of $1+r_{X}(1)$ With other GeOMetric COefFicients}

The following example shows that $1+r_{X}(1)$ can be greater than $\kappa_{\omega}(X)$.

Example 4.1. Let $X=l_{2} \oplus \mathbb{R}$ be equipped with a norm

$$
\|(x, r)\|=\|x\|_{2}+\max \left\{|r|-\frac{1}{2}\|x\|_{2}, 0\right\},
$$

where $x \in l_{2}, r \in \mathbb{R}$ and $\|\cdot\|_{2}$ is the usual $l_{2}$ norm. The space $X$ has the nonstrict Opial property. More careful calculation than in [28] shows $1+r_{X}(1)=\frac{5}{4}$. Next, it is easy to observe that $W C S(X)=w-S O C(X)=\sqrt{2}$ and $\sqrt{W C S(X)}=$ $\sqrt{w-S O C(X)}<\frac{5}{4}$. Now we compute $\kappa_{\omega}(X)$. Let us take arbitrary $1<a<b$. For sufficiently small $0<\epsilon<1$ the numbers

$$
a \cdot(1-\epsilon)-\frac{1}{2} \quad \text { and } \quad b \cdot(1-\epsilon)-\frac{1}{2}
$$

are strictly greater than $\frac{1}{2}$. Let $y_{n}=\left(e_{n}, 0\right)$ for $n=1,2, \ldots$, where $\left\{e_{n}\right\}$ is the standard basis in $l_{2}$. For $r=1-\epsilon$ and

$$
x=\left(0, \frac{1}{2}-a \cdot(1-\epsilon)\right), \quad y=\left(0, b \cdot(1-\epsilon)-\frac{1}{2}\right)
$$

we get $\|x-y\|>1>r, \lim _{n \rightarrow \infty}\left\|y_{n}-y\right\|=b r, \lim _{n \rightarrow \infty}\left\|y_{n}-x\right\|=$ ar and $\lim _{n \rightarrow \infty}\left\|y_{n}\right\|=1>r$. All these facts imply $\kappa_{\omega}(X)=1$. 
The next example shows that the coefficient $\sqrt{W C S(X)}$ is better in some cases than $1+r_{X}(1)$.

Example 4.2. In a Banach space $X$ with the uniform Opial condition [25] (i.e. for each $c>0$, there exists $r>0$ such that

$$
\liminf _{n \rightarrow \infty}\left\|\xi+\xi_{n}\right\| \geq 1+r
$$

for each $\xi \in X$ with $\|\xi\| \geq c$ and all weakly null sequences $\left\{\xi_{n}\right\}$ in $X$ such that $\liminf _{n \rightarrow \infty}\left\|\xi_{n}\right\| \geq 1$ ) the coefficient $\kappa_{\omega}(X)$ is equal to $1+r_{X}(1)$ (see [8]). As it was shown in [6] the space $X=l_{2}$ renormed by

$$
\|x\|=\left[\left|x_{1}\right|^{p}+\left(\sum_{n=2}^{\infty}\left|x_{n}\right|^{2}\right)^{\frac{p}{2}}\right]^{\frac{1}{p}},
$$

where $x=\left(x_{n}\right) \in l^{2}$ and $2<p<\infty$, has the uniform Opial property, $\kappa_{\omega}(X)=$ $1+r_{X}(1) \leq 2^{\frac{1}{p}}$ and $W C S(X)=w-S O C(X)=\sqrt{2}$. For sufficiently large $p$ we get $\kappa_{\omega}(X)<\sqrt{W C S(X)}=\sqrt{w-S O C(X)}$.

\section{FINAL REMARKS}

Remark 5.1. The space from Example 4.1 was suggested to H.-K. Xu by P.-K. Lin [28] as the space which has $c_{0}(X)=\sup \left\{c \geq 0: r_{X}(c) \leq 0\right\}<1$ but does not have Opial's property.

Remark 5.2. Generally the coefficient $r_{X}(1)$ seems to be easier for computation than $\kappa_{\omega}(X)$. As we see above $\kappa_{\omega}(X)$ can be strictly less then $1+r_{X}(1)$. Does there exist a Banach space $X$ with $1+r_{X}(1)<\kappa_{\omega}(X)$ ?

Remark 5.3. The simple analysis of the proof of Theorem 2.2 in [8] gives the following result.

Theorem 5.1. For every Banach space $X$ we have

$$
\begin{aligned}
1+r_{X}(1)= & \sup \{b>0: \\
& \forall_{r>0} \forall_{x, y \in X} \forall\left\{x_{n}\right\} \subset X\left[\left(\|x-y\| \geq r \wedge x_{n} \rightarrow x \wedge\right.\right. \\
& \left.\left.\left.\wedge \limsup _{n \rightarrow \infty}\left\|y-x_{n}\right\| \leq b r\right) \Longrightarrow \liminf _{n \rightarrow \infty}\left\|x-x_{n}\right\| \leq r\right]\right\} .
\end{aligned}
$$

\section{REFERENCES}

[1] BAILLON J.-B., Quelques aspects de la théorie des points fixes dans les espaces de Banach I, Séminaire d'Analyse Fonctionnelle de l'Ecole Polytechnique VII (1978-1979). MR 81d:47036

[2] BROWDER F.E. \& PETRYSHYN W.V., The solution by iteration of nonlinear functional equations in Banach spaces, Bull. Amer. Math. Soc. 72 (1966), 571-576. MR 32:8155b

[3] M. BUDZYŃSKA, T. KUCZUMOW \& S. REICH, A uniformly asymptotic normal structure, a semi-Opial property of Banach spaces and fixed points of uniformly lipschitzian semigroups. Part I, Abstr. Appl. Anal. (to appear).

[4] M. BUDZYŃSKA, T. KUCZUMOW \& S. REICH, A uniformly asymptotic normal structure, a semi-Opial property of Banach spaces and fixed points of uniformly lipschitzian semigroups. Part II, Abstr. and Appl. Anal. (to appear).

[5] BYNUM W. L., Normal structure coefficients for Banach spaces, Pacif. J. Math. 86 (1980), 427-436. MR 81m:46030 
[6] DOMÍNGUEZ BENAVIDES T., Fixed point theorems for uniformly Lipschitzian mappings and asymptotically regular mappings, Nonlinear Analysis 32 (1998), 15-27. CMP 98:07

[7] DOMÍNGUEZ BENAVIDES T., Stability of the fixed point property for nonexpansive mappings, preprint.

[8] DOMÍNGUEZ BENAVIDES T. \& XU H.-K., A new geometrical coefficient for Banach spaces and its applications in fixed point theory, Nonlinear Analysis 25 (1995), 311-325. MR 96e: 47062

[9] EDELSTEIN M. \& O'BRIEN R.C., Nonexpansive mappings, asymptotic regularity, and successive approximations, J. London Math. Soc. 17 (1978), 547-554. MR 80b:47074

[10] GOEBEL K. \& KIRK W.A., A fixed point theorem for transformations whose iterates have uniform Lipschitz constant, Studia Math. 47 (1973), 135-140. MR 49:1242

[11] GOEBEL K. \& KIRK W.A., Iteration processes for nonexpansive mappings,, Topological Methods in Nonlinear Functional Analysis ( S.P. Singh and S. Thomeier, Eds), Contemporary Mathematics, American Mathematical Society, Providence, RI, vol. 21, 1983, pp. 115-123. MR 85a:47059

[12] GOEBEL K. \& KIRK W.A., Topics in metric fixed point theory, Cambridge University Press, 1990. MR 92c: 47070

[13] GÓRNICKI J., Fixed points of asymptotically regular semigroups in Banach spaces, Rend. Circ. Mat. Palermo 46 (1997), 89-118. MR 98f:47064

[14] GÓRNICKI J. \& KRÜPPEL M., Fixed points of uniformly lipschitzian mappings, Bull. Polish Acad. Sci. Math. 36 (1988), 57-63. MR 91a:47079

[15] ISHIKAWA S., Fixed points and iteration of a nonexpansive mapping in a Banach space, Proc. Amer. Math. Soc. 59 (1976), 65-71. MR 54:1030

[16] JIMÉNES-MELADO A. \& LLORENS-FUSTER E., Stability of the fixed point property for nonexpansive mappings, Houston J. of Math. 18 (1992), 251-257.

[17] JIMÉNES-MELADO A. \& LLORENS-FUSTER E., Opial modulus and stability of the fixed point property, preprint.

[18] KIM T.H. \& KIRK W.A., Fixed point theorems for Lipschitzian mappings in Banach spaces, Nonlinear Analysis 26 (1996), 1905-1911. MR 97b:47061

[19] KRASNOSELSKII M.A., Two observations about the method of successive approximations, Uspehi Mat. Nauk 10 (1955), 123-127. MR 16:833a

[20] LIM T.-C. \& XU H.-K., Uniformly Lipschitzian mappings in metric spaces with uniform normal structure, Nonlinear Analysis 25 (1995), 1231-1235. MR 96i:54039

[21] LIN P.-K., A Uniformly asymptotically regular mapping without fixed points, Canad. Math. Bull. 30 (1987), 481-483. MR 89a:47080

[22] LIN P.-K., Stability of the fixed point property of Hilbert spaces, preprint.

[23] LIN P.-K., TAN K.-K. \& XU H.-K., Demiclosedness principle and asymptotic behavior for asymptotically nonexpansive mappings, Nonlinear Analysis 24 (1995), 929-946. MR 96a:47094

[24] OPIAL Z., Weak convergence of the sequence of successive approximations for nonexpansive mappings, Bull. Amer. Math. Soc. 73 (1967), 591-597. MR 35:2183

[25] PRUS S., Banach spaces with the uniform Opial property, Nonlinear Analysis 18 (1992), 697-704. MR 93h:46022

[26] TINGLEY D., Noncontractive uniformly lipschitzian semigroups in Hilbert space, Proc. Amer. Math. Soc. 92 (1984), 355-361. MR 85k:47105

[27] TINGLEY D., An asymptotically nonexpansive commutative semigroup with no fixed points, Proc. Amer. Math. Soc. 97 (1986), 107-113. MR 87g:47113

[28] XU. H.-K., Geometrical coefficients of Banach spaces and nonlinear mappings, Recent Advances on metric fixed point theory (T. Domínguez Benavides, ed.), Universidad de Sevilla, Serie: Ciencias, Núm. 48 (1996), 161-178. MR 98f:46012

Instytut Matematyki, UMCS, 20-031 Lublin, Poland

E-mail address: tadek@golem.umcs.lublin.pl 